\title{
The Impacts of Childhood Trauma on Psychosocial Features in a Chinese Sample of Young Adults
}

\author{
Dandan Wang1, Shaojia Lu' ${ }^{1}$, Weijia Gao², Zhaoguo Wei ${ }^{3,4}$, Jinfeng Duan', \\ Shaohua $\mathrm{Hu}^{1}$, Manli Huang ${ }^{1}$, Yi Xu' ${ }^{1}$, and Lingjiang $\mathrm{Li}^{3 凶}$ \\ 1'Department of Psychiatry, The First Affiliated Hospital, Zhejiang University School of Medicine, Key Laboratory of Mental Disorder's \\ Management of Zhejiang Province, Hangzhou, China \\ 2Department of Child Psychology, The Children's Hospital, Zhejiang University School of Medicine, Hangzhou, China \\ ${ }^{3}$ Mental Health Institute of The Second Xiangya Hospital, Key Laboratory of Psychiatry and Mental Health of Hunan Province, \\ Central South University, Changsha, China \\ ${ }^{4}$ Department of Psychiatry, Shenzhen Kangning Hospital, Shenzhen, China
}

Objective The aims of the present study were to explore the occurrence of childhood trauma and importantly to determine the impacts of childhood trauma on psychosocial features in a Chinese sample of young adults.

Methods A survey was carried out in a group of 555 university students by using Childhood Trauma Questionnaire (CTQ), Self-rating Depression Scale (SDS), Self-rating Anxiety Scale (SAS), Dysfunctional Attitudes Questionnaire (DAS), Eysenck Personality Questionnaire (EPQ), and Social Support Rating Scale (SSRS). The moderate-severe cut-off scores for CTQ were used to calculate the prevalence of childhood trauma, and then psychosocial features were compared between individuals with and without childhood trauma.

Results A proportion of $18.6 \%$ of university students had self-reported childhood trauma exposures. Subjects with childhood trauma reported higher scores of SDS, SAS, DAS, and psychoticism and neuroticism dimensions of EPQ $(t=4.311-5.551, p<0.001)$; while lower scores of SSRS and extraversion dimension of EPQ $(\mathrm{t}=-4.061--3.039, \mathrm{p}<0.01)$. Regression analyses further revealed that scores of SAS and DAS were positively (Adjusted $\mathrm{B}=0.211-0.230, \mathrm{p}<0.05$ ), while scores of SSRS were negatively (Adjusted $\mathrm{B}=-0.273--0.240$, $\mathrm{p}<0.05$ ) associated with specific CTQ scores.

Conclusion Childhood trauma is still a common social and psychological problem. Individuals with childhood trauma show much more depression, anxiety, distorted cognition, personality deficits, and lower levels of social support, which may represent the social and psychological vulnerability for developing psychiatric disorders after childhood trauma experiences.

Psychiatry Investig 2018;15(11):1046-1052

Key Words Childhood trauma, Depression and anxiety, Dysfunctional attitude, Personality trait, Social support.

\section{INTRODUCTION}

Childhood trauma is the experience of a single or multiple events by a child that is emotionally painful or distressful, which often leads to seriously lifelong damages to physical

Received: April 9, 2018 Revised: July 16, 2018

Accepted: September 26, 2018

$\triangle$ Correspondence: Shaojia Lu, MD

Department of Psychiatry, The First Affiliated Hospital, Zhejiang University School of Medicine, No. 79 Qingchun Road, Hangzhou 310003, China Tel: +86 571 87235948, Fax: +86 571 87235948, E-mail: 1314004@zju.edu.cn

$\triangle$ Correspondence: Lingjiang Li, MD

Mental Health Institute of The Second Xiangya Hospital, Central South University, No. 139 Renmin Road, Changsha 410011, China

Tel: +86 731 85295413, Fax: +86 731 85295413, E-mail: 1lj2920@163.com

(c) This is an Open Access article distributed under the terms of the Creative Commons Attribution Non-Commercial License (http://creativecommons.org/licenses/bync/4.0) which permits unrestricted non-commercial use, distribution, and reproduction in any medium, provided the original work is properly cited. and mental health. ${ }^{1}$ Childhood trauma has became a major social public-health problem worldwide. Every year, in highincome countries, about $4-16 \%$ of children are experiencing physical abuse and one in ten is neglected or psychologically abused. ${ }^{2}$ In China, a recent meta analysis indicated that the estimated prevalence of physical abuse, emotional abuse, sexual abuse, and neglect in children under 18 years of age were $26.6 \%, 19.6 \%, 8.7 \%$, and $26 \%$, respectively. ${ }^{3}$ In this context, childhood trauma also represents a common and significant public health burden, and it is found that in reported maltreated cases there is an estimated $\$ 124$ billion annual burden on the US health-care system. ${ }^{4}$

Over the past decades, extensive studies suggested that childhood trauma contributed to an increased risk of diverse mental disorders that continued into adulthood, ${ }^{5}$ however, the potential mechanisms underlying this association remains 
unclear and complicated. In recent years, plenty of studies have enriched our understanding of the psychological sequelae of childhood trauma in several aspects. For instance, previous studies have demonstrated that childhood trauma has strong effects on later psychological distress, such as depression and anxiety traits. ${ }^{6}$ Simultaneously, personality deficits and maladaptive interpersonal styles were also reported to be associated with childhood trauma, ${ }^{7}$ and neuroticism was confirmed as one of the psychological processes in which childhood trauma played a role in the development of psychiatric disorders. ${ }^{8}$ Furthermore, it is elucidated that childhood trauma can lead to dysfunctional attitudes causing negative cognitive structuring and mistakes in data processing. In a preceding study, a negative correlation was found between dysfunctional attitudes and childhood emotional abuse in female patients with depressive disorder, which might be interpreted as that emotional abuse could be effective in development of dysfunctional attitudes, causing victims to be prone to depression. ${ }^{9}$

Social support can be a protective factor in maintaining mental wellbeing. Nevertheless, exposures to traumatic events during childhood are generally associated with low levels of social support in adulthood..$^{10}$ Patients with mental disorders, such as depression, bipolar disorder, and schizophrenia, were found to experience more severe childhood trauma and poorer social support than normal controls, ${ }^{11}$ which advocated the indication that social support might function as a mediator in the association between childhood trauma and later psychopathology. ${ }^{12}$ On the contrary, high levels of social support was identified as a resilience factor against the negative effect of adverse childhood experiences on health outcomes in adulthood. ${ }^{13}$

As listed above, prior studies have proved that childhood trauma may result in a series of deleterious outcomes of social and psychological characteristics which could be served as mediators between childhood trauma and later psychopathology. However, the majority of these studies were conducted in psychiatric patients, which raised questions concerning potential sample and selection bias. In addition, most studies were proceeded in western countries, relatively few studies have been developed to examine the association between childhood trauma and adulthood psychosocial features in Asian countries $^{14-16}$ and they mainly concentrate on limited aspects of psychopathology outcomes, such as depressive symptoms or personality deficits. Interestingly, the cross-cultural variations in psychosocial development have been substantiated in previous studies. ${ }^{17}$ Under these circumstances, general population studies investigating the impacts of childhood trauma on various psychosocial characteristics are needed, particularly in Chinese samples.
Hence, the aims of the present study were to explore the occurrence of childhood trauma and importantly to determine the impacts of childhood trauma on psychosocial features, including levels of anxiety and depression, personality traits, dysfunctional attitudes and social support, in a Chinese sample of young adults.

\section{METHODS}

\section{Participants}

In this study, a total of 555 students (male/female, 101/454), from a local university were recruited via advertisements posted in the campus. This university is a four-year undergraduate-level normal university located in Changsha city, which has over 16,000 undergraduates. Changsha, the capital city of Hunan province, is a new first-tier city in the middle of China. Data were collected during October to November, 2011. All the participants were freshmen from different departments of the university and they responded with no direct reference to childhood trauma as a key variable. The average age of the male and female subjects were 19.2 \pm 1.75 and 19.0 \pm 2.00 years, respectively. The principal investigator obtained an approval to conduct this study. This study was approved by the ethic committee of the Second Xiangya Hospital of Central South University (2011-S049).

\section{Procedures}

The investigators received the volunteers and provided them with a detailed description of the purpose and content of the present study. Students were informed that they needed to finish seven questionnaires in 45 minutes in the classroom of the university and that they were free to quit at any time. Issues of anonymity and confidentiality were discussed and consent to participate was implied by the return of a written informed consent. All participants were asked to answer the questionnaires independently and to not discuss with others. The completed questionnaires were handed in to the investigators directly.

\section{Instruments}

A battery of questionnaires were administered for the needs of this study, including general information, Childhood Trauma Questionnaire (CTQ), Self-rating Depression Scale (SDS), Self-rating Anxiety Scale (SAS), Dysfunctional Attitudes Scale (DAS), Eysenck Personality Questionnaire (EPQ), and Social Support Rating Scale (SSRS).

The CTQ is a 28-item retrospective self-report questionnaire designed to evaluate five types of adverse childhood experiences by five subscales: emotional neglect, emotional abuse, sexual abuse, physical neglect, and physical abuse, re- 
spectively. Individuals who score higher than the moderatesevere threshold of a subscale are treated as existence of corresponding childhood trauma experience. The cutoffs of each subscale are 1) emotional abuse $\geq 13,2$ ) emotional neglect $\geq 15,3$ ) sexual abuse $\geq 8,4$ ) physical abuse $\geq 10$, and 5) physical neglect $\geq 10$, which have provided good sensitivity and specificity for confirmed abuse or neglect. ${ }^{18}$ The Chinese version of CTQ was used in our study, which was revealed to have good internal consistency (Cronbach's $\alpha$ ) for the CTQ total score $(0.77)$ and the five subscales $(0.41-0.78)$ in a Chinese sample. ${ }^{19}$

Both SDS $^{20}$ and SAS $^{21}$ are 20-item and self-report scales. Each item of the two scales is rated on a 4-point grade scale that ranges from $1=$ none or a little of the time to $4=$ most or all of the time. The SDS measures the severity of depressive symptoms while the SAS assesses anxiety symptoms. The Chinese version of SDS and SAS are widely used and have adequate reliability (Cronbach's a values of SDS and SAS were 0.862 and 0.931 , respectively). ${ }^{22,23}$

The DAS ${ }^{24}$ is a 40 -item scale designed to assess cognitive vulnerability to depression, which may reflect the impacts of early adverse events on one's cognition about self and the world. Each item ranks from $1=$ completely disagree to $7=$ completely agree. Higher scores reflect more dysfunctional attitudes. The Chinese version was introduced to our study, which displays a good reliability (Cronbach's $\alpha=0.87){ }^{25}$

The EPQ is designed to measure personality dimensions. ${ }^{26}$ The 48-item, revised, short scale for Chinese was used in the present study. It can be divided into 4 subscales, evaluating neuroticism $(\mathrm{N})$, extraversion $(\mathrm{E})$, psychoticism $(\mathrm{P})$, and Lie (L) dimensions, respectively, and each subscale has 12 items. The Chinese version shows good internal consistency (Cronbach's $\alpha$ ) for the four subscales (0.54-0.75) ${ }^{27}$

The SSRS ${ }^{28}$ is a 10 -item, self-rating inventory in Chinese, which is designed by Xiao and has been widely used in Chinese studies. The questionnaire measures social support on three aspects, subjective support, objective support, and utilization of social services. The SSRS shows good internal consistency (Cronbach's $\alpha$ ) for the total score $(0.90)$ and the three subscales $(0.83-0.85) .^{29}$

\section{Statistical analysis}

All statistical analyses were conducted using Statistical Package for the Social Sciences (SPSS) version 16.0 (SPSS Inc., Chicago, IL, USA). Independent two-sample t tests and chi-square tests $\left(\chi^{2}\right)$ were respectively used to tests for the continuous variables and categorical variables between two groups. Values are given as mean \pm standard deviation. The level of twotailed statistical significance was set at $\mathrm{p}<0.05$ for all tests. Several stepwise multiple linear regression models were used to identify and quantify the relationships of psychosocial features with CTQ scores. The stepping criteria employed for entry and removal were based on the significance level of the F-value and set at 0.05 and 0.10 , respectively.

\section{RESULTS}

\section{The prevalence of childhood trauma}

A total of 103 out of 555 university students had self-reported childhood trauma exposures, the prevalence rate was $18.6 \%$, a proportion of $42.7 \%(n=44)$ of maltreated individuals experienced at least two forms of childhood trauma. Physical neglect ( $n=66,11.9 \%$ ) was the most common aspect of childhood trauma followed by emotional neglect, sexual abuse, emotional abuse, and physical abuse (Figure 1). The prevalence of each type of childhood trauma did not differ in sex in our sample, the detailed descriptions were summarized in Table 1.

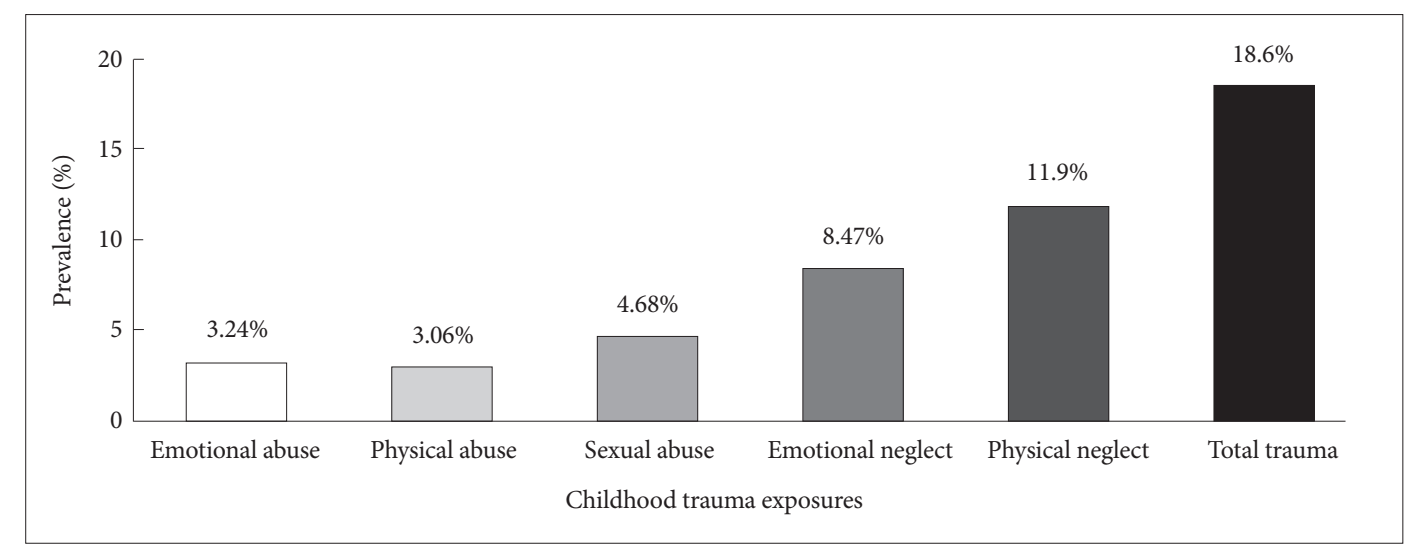

Figure 1. The prevalence of childhood trauma in the present sample. The prevalence of childhood trauma in our sample was $18.6 \%$. The prevalence of five types of childhood trauma ranked as follows, physical neglect (11.9\%), emotional neglect $(8.47 \%)$, sexual abuse $(4.68 \%)$, emotional abuse (3.24\%), and physical abuse (3.06\%). 
Table 1. The prevalence of five types of childhood trauma in male and female students

\begin{tabular}{lrccc}
\hline & \multicolumn{2}{c}{ Gender } & \multirow{2}{*}{$\chi^{2}$} & p-value \\
\cline { 2 - 3 } & \multicolumn{1}{c}{ Male } & Female & & \\
\hline Total trauma, N (\%) & $20(19.8)$ & $83(18.3)$ & 0.126 & 0.722 \\
Emotional abuse & $5(4.95)$ & $13(2.86)$ & 1.150 & 0.284 \\
Physical abuse & $3(2.97)$ & $14(3.08)$ & 0.004 & 0.952 \\
Sexual abuse & $5(4.95)$ & $21(4.63)$ & 0.020 & 0.889 \\
Emotional neglect & $10(9.90)$ & $37(8.15)$ & 0.327 & 0.568 \\
Physical neglect & $13(12.9)$ & $53(11.7)$ & 0.113 & 0.737 \\
\hline
\end{tabular}

Table 2. The comparisons of social and psychological characteristics between individuals with and without childhood trauma

\begin{tabular}{|c|c|c|c|c|}
\hline & $\begin{array}{l}\text { Childhood } \\
\text { trauma group, } \\
\mathrm{N}=103, \\
\text { mean }(\mathrm{SD})\end{array}$ & $\begin{array}{l}\text { Non-childhood } \\
\text { trauma group, } \\
\mathrm{N}=452 \text {, } \\
\text { mean }(\mathrm{SD})\end{array}$ & $\mathrm{t}$ & p-value \\
\hline SDS & $35.3(7.68)$ & $31.6(6.28)$ & 5.183 & 0.000 \\
\hline SAS & $32.2(5.83)$ & $29.4(5.25)$ & 4.650 & 0.000 \\
\hline DAS & $125.7(25.0)$ & $113.3(19.2)$ & 5.551 & 0.000 \\
\hline EPQ-P & $2.91(2.10)$ & $2.18(1.41)$ & 4.311 & 0.000 \\
\hline EPQ-E & $7.74(3.35)$ & $8.71(2.92)$ & -2.949 & 0.003 \\
\hline EPQ-N & $5.40(3.45)$ & $3.77(3.13)$ & 4.677 & 0.000 \\
\hline EPQ-L & $4.54(2.65)$ & $5.48(2.56)$ & -3.340 & 0.001 \\
\hline SSRS & $39.7(9.02)$ & $42.7(9.00)$ & -3.039 & 0.002 \\
\hline OS & $7.05(2.01)$ & $7.92(1.95)$ & -4.061 & 0.000 \\
\hline SS & $24.8(8.45)$ & $26.2(8.47)$ & -1.480 & 0.139 \\
\hline UOS & $7.83(1.93)$ & $8.59(1.73)$ & -3.982 & 0.000 \\
\hline
\end{tabular}

DAS: dysfunctional attitudes scale, E: extraversion, EPQ: eysenck personality questionnaire, L: lie, N: neuroticism, OS: objective support, P: psychoticism, SAS: self-rating anxiety scale, SD: standard deviation, SDS: self-rating depression scale, SS: subjective support, SSRS: social support rating scale, UOS: utilization of social services

\section{The comparisons of psychosocial features}

As compared with subjects without childhood trauma, individuals with adverse childhood experiences reported higher scores of SDS, SAS, DAS, and P and N dimensions of EPQ, while lower scores of $\mathrm{E}$ dimension of $\mathrm{EPQ}$, and SSRS, including scores of objective support and utilization of social services, all $\mathrm{p}<0.05$, for more details, please refer to Table 2 .

\section{Linear regression analyses}

As listed in Table 3, the multiple linear regression analyses revealed that, in subjects with childhood trauma, scores of SAS were positively associated with severity of physical neglect (adjusted $\mathrm{B}=0.230, \mathrm{p}=0.02$ ); scores of DAS were positively correlated with severity of sexual abuse (adjusted $\mathrm{B}=0.211, \mathrm{p}=$ 0.033); and SSRS total scores were negatively associated with severity of emotional abuse (adjusted $\mathrm{B}=-0.273, \mathrm{p}=0.005$ ), in
Table 3. Stepwise multiple linear regressions analyses: associations of social and psychological characteristics with childhood trauma

\begin{tabular}{|c|c|c|c|c|}
\hline & Adjusted B & SE & $\mathrm{t}$ & $\mathrm{p}$-value \\
\hline SAS & & 0.153 & 2.370 & 0.020 \\
\hline Physical neglect & 0.230 & & & \\
\hline Adjusted $\mathrm{R}^{2}$ & 0.043 & & & \\
\hline F-change & 5.617 & & & \\
\hline p-value (F-change) & 0.020 & & & \\
\hline DAS & & 1.072 & 2.167 & 0.033 \\
\hline Sexual abuse & 0.211 & & & \\
\hline Adjusted $\mathrm{R}^{2}$ & 0.035 & & & \\
\hline F-change & 4.694 & & & \\
\hline p-value (F-change) & 0.033 & & & \\
\hline SSRS & & 0.224 & -2.853 & 0.005 \\
\hline Emotional abuse & -0.273 & & & \\
\hline Adjusted $\mathrm{R}^{2}$ & 0.065 & & & \\
\hline F-change & 8.137 & & & \\
\hline p-value (F-change) & 0.005 & & & \\
\hline SSRS-OS & & 0.051 & -2.489 & 0.014 \\
\hline Physical neglect & -0.240 & & & \\
\hline Adjusted $\mathrm{R}^{2}$ & 0.048 & & & \\
\hline F-change & 6.193 & & & \\
\hline p-value (F-change) & 0.014 & & & \\
\hline
\end{tabular}

DAS: dysfunctional attitudes scale, OS: objective support, SAS: Selfrating anxiety scale, SE: standard error, SSRS: social support rating scale

particular, scores of objective support were adversely correlated with severity of physical neglect (adjusted $\mathrm{B}=-0.240, \mathrm{p}=$ 0.014). However, no association was found between CTQ scores and scores of SDS and EPQ.

\section{DISCUSSION}

The current study investigated the prevalence of childhood trauma and the impacts of childhood trauma on psychosocial features in a general population of university students. Our results indicated a high prevalence of childhood trauma in such a sample, and furthermore, we demonstrated a negative effect of childhood trauma on social and psychological development. Our findings may have potential significance for contributing to understanding the social and psychological mechanisms of early life stress.

In this study, the prevalence rate of childhood trauma was $18.6 \%$, with five types of trauma ranged from $3.06 \%$ to $11.9 \%$, which may suggest that childhood trauma is a common problem. However, the prevalence of five types of childhood trauma were much higher in a previous comparable Chinese 
study, ${ }^{30}$ this may be mainly explained by the respective different cutoffs on the subscales of CTQ. The moderate-severe thresholds used in our study were higher than those in aforementioned study, making the rates of the subscales lower in comparisons. Moreover, the disaccord can also be the result of different measures of childhood trauma. ${ }^{31}$ We further found that physical neglect was most commonly reported by participants, together with prior reports, ${ }^{16}$ these findings indicate that child neglect is a more common form of childhood maltreatment. The present study did not find any sex difference on prevalence of childhood trauma, which is not in accordance with previous studies. ${ }^{32,33}$ We speculate that the gender imbalance in our sample may be partially responsible for this inconsistent finding. Taken together, it is acknowledged that prevalence rates of childhood trauma vary considerably with the method used for assessment.

A second noteworthy finding of the current study was elevated levels of depression and anxiety in subjects with childhood trauma. In nonclinical community volunteers, exposure to a greater number of early life stressor events has been shown to account for significantly higher levels of self-reported depressive and anxiety symptoms. ${ }^{34}$ Women with childhood sexual abuse often suffered from psychiatric symptoms, including depression and anxiety, as measured by Symptom Checklist-90 (SCL-90). ${ }^{35}$ It has also been revealed that healthy adults with low parental care experiences exhibit decreased self-esteem, and increased depressive symptomatology and anxiety. ${ }^{36}$ Additionally, in a prior meta-analysis, sexual and physical abuse in childhood was demonstrated to be associated with high levels of depression, anxiety and distress over the life course. ${ }^{37}$ Our results are generally consistent with these findings, which may suggest a pathway that links early life trauma and depression and anxiety disorders in adulthood.

In the present study, individuals with childhood trauma showed increased scores of DAS which suggested more dysfunctional attitudes. Cognitive theories have identified different cognitive vulnerabilities and processes hypothesized to predict the onset and/or maintenance of depression, while Beck's theory focuses on negative schema and dysfunctional attitudes. ${ }^{38}$ Maltreatment, such as abuse or neglect, is deemed to act as a severe environmental risk that may contribute to the development of cognitive vulnerabilities. ${ }^{39}$ Among adolescents, greater stress levels have been reported to predict more dysfunctional attitudes. ${ }^{40}$ Of note, women with childhood sexual abuse were observed to hold more dysfunctional beliefs, as measured by DAS, in a non-clinical sample. ${ }^{41}$ Similarly, we also found a relationship between sexual abuse and dysfunctional attitudes in traumatic subjects, which may highlight the importance of early sexual abuse in predicting dysfunctional thoughts in adulthood. Interestingly, previous stud- ies have suggested that childhood trauma may increase the risk to depression in later life through affecting the development of dysfunctional attitudes in maltreated individuals. ${ }^{9}$ Thus, our present finding added to the literature on elucidating the psychological link between childhood trauma and depression.

Moreover, this study confirmed an adverse effect of childhood trauma on personality development. Individuals subjected to childhood trauma had significantly higher EPQ-P and EPQ-N scores, but lower scores of EPQ-E. This is partially consistent with a Chinese study that showed abused adolescents had higher EPQ-P and EPQ-N scores than the control group. ${ }^{16}$ In addition, the relationship of childhood trauma with increased levels of psychoticism and neuroticism were revealed in clinical samples as well. ${ }^{42,43}$ In contrast, our study further found a decreased EPQ-E score in maltreated subjects. Extraversion is prone to experience positive life events, which may serve as resilience for psychological distress. ${ }^{44}$ The current outcome is in line with a previous finding that lower extraversion scores were observed in women who had been sexually abused by a parent. ${ }^{45}$ Overall, plenty of studies have indicated that personality is a main factor influencing mental health, ${ }^{46}$ hence, our findings suggest the possibility that childhood trauma may predispose to mental disorders by determining the EPQ-P, EPQ-N, and EPQ-E.

Finally, the present study indicated that experiences of childhood trauma were related to decreased levels of social support, including objective support and utilization of social services. This indication is supported by a number of prior studies. ${ }^{10,47}$ Low-level social support is a well known risk factor of developing mental disorders and unfavorable behavior patterns, for instance, major depressive disorder (MDD), schizophrenia, post-traumatic stress disorder (PTSD), and suicidal attempts, ${ }^{48-50}$ which are closely correlated with childhood trauma. Further studies also confirmed a moderating effect of social support on the association between childhood trauma and symptoms of PTSD and MDD in adulthood .

\section{Limitation}

Several limitations of the present study need to be acknowledged. Firstly, although the participants were recruited from different departments of a university, our current sample might be not sufficiently representative. The limited sample size might increase the chance of sampling bias. Also, the gender imbalance in our sample might generate bias for the prevalence of childhood trauma. Secondly, demographic data, such as growth environment and family status were not collected in this study, which restricted us to explore the risk factors of developing childhood trauma. Thirdly, since the proportion of multiple trauma exposures in our sample were high, 
we did not investigate the effects of different types of childhood trauma on social and psychological alterations. Fourthly, this study was a cross-sectional design, which precluded causal inferences. Meanwhile, data on childhood trauma were obtained by a retrospective questionnaire, which might lead to information bias. Finally, all the questionnaires used in this study were self-rating scales, instead of structured or semistructured interviews, the outcomes might be influenced by subjectivity of participants. In this context, the present findings need to be replicated in a larger and better designed study.

In conclusion, the current study suggests that childhood trauma is still a common social and psychological problem. Individuals with childhood trauma show much more depression, anxiety, distorted cognition, personality deficits, and lower levels of social support, which may represent the social and psychological vulnerability for developing psychiatric disorders after childhood trauma experiences.

\section{Acknowledgments}

We sincerely thank the support of funds from the National Natural Science Foundation of China (81601182 to Shaojia Lu), and Key Research Project of Zhejiang Province (2015C03040 to Yi Xu).

\section{REFERENCES}

1. Pechtel P, Pizzagalli DA. Effects of early life stress on cognitive and affective function: an integrated review of human literature. Psychopharmacology (Berl) 2011;214:55-70.

2. Gilbert R, Widom CS, Browne K, Fergusson D, Webb E, Janson S. Burden and consequences of child maltreatment in high-income countries. Lancet 2009;373:68-81.

3. Fang X, Fry DA, Ji K, Finkelhor D, Chen J, Lannen P, et al. The burden of child maltreatment in China: a systematic review. Bull World Health Organ 2015;93:176C-185C.

4. Buckingham ET, Daniolos P. Longitudinal outcomes for victims of child abuse. Curr Psychiatry Rep 2013;15:342.

5. Takizawa R, Maughan B, Arseneault L. Adult health outcomes of childhood bullying victimization: evidence from a five-decade longitudinal British birth cohort. Am J Psychiatry 2014;171:777-784.

6. Ekinci S, Kandemir H. Childhood trauma in the lives of substance-dependent patients: the relationship between depression, anxiety and self-esteem. Nord J Psychiatry 2015;69:249-253.

7. Allen B, Lauterbach D. Personality characteristics of adult survivors of childhood trauma. J Trauma Stress 2007;20:587-595.

8. Roy A. Childhood trauma and neuroticism as an adult: possible implication for the development of the common psychiatric disorders and suicidal behaviour. Psychol Med 2002;32:1471-1474.

9. Akbaba Turkoglu S, Essizoglu A, Kosger F, Aksaray G. Relationship between dysfunctional attitudes and childhood traumas in women with depression. Int J Soc Psychiatry 2015;61:796-801.

10. Sperry DM, Widom CS. Child abuse and neglect, social support, and psychopathology in adulthood: a prospective investigation. Child Abus Negl 2013;37:415-425.

11. Xie P, Wu K, Zheng Y, Guo Y, Yang Y, He J, et al. Prevalence of childhood trauma and correlations between childhood trauma, suicidal ideation, and social support in patients with depression, bipolar disorder, and schizophrenia in southern China. J Affect Disord 2018;228: 41-48.

12. Salazar AM, Keller TE, Courtney ME. Understanding social support's role in the relationship between maltreatment and depression in youth with foster care experience. Child Maltreat 2011;16:102-113.

13. Logan-Greene P, Green S, Nurius PS, Longhi D. Distinct contributions of adverse childhood experiences and resilience resources: a cohort analysis of adult physical and mental health. Soc Work Health Care 2014;53:776-797.

14. Kim SS, Jang H, Chang HY, Park YS, Lee DW. Association between childhood adversities and adulthood depressive symptoms in South Korea: results from a nationally representative longitudinal study. BMJ Open 2013; 3. pii: e002680.

15. Takeuchi H, Hiroe T, Kanai T, Morinobu S, Kitamura T, Takahashi K, et al. Childhood parental separation experiences and depressive symptomatology in acute major depression. Psychiatry Clin Neurosci 2003; 57:215-219.

16. Li X, Wang Z, Hou Y, Wang Y, Liu J, Wang C. Effects of childhood trauma on personality in a sample of Chinese adolescents. Child Abus Negl 2014;38:788-796.

17. Zhao S, Zhang J. The association between depression, suicidal ideation and psychological strains in college students: a cross-national study. Culture, medicine and psychiatry. 2018 [Epub ahead of print].

18. Bernstein D, Fink L. Childhood Trauma Questionnaire: A Retrospective Self-Report. San Antonio, TX: The Psychological Corporation; 1998.

19. Zhao X, Zhang Y, Li L, Zhou Y, Li H, Yang S. Reliability and validity of the Chinese version of Childhood Trauma Questionnaire. Chin J Clin Rehabil 2005;9:105-107.

20. Zung WW, Richards CB, Short MJ. Self-rating depression scale in an outpatient clinic. Further validation of the SDS. Arch Gen Psychiatry 1965;13:508-515.

21. Zung WW. A rating instrument for anxiety disorders. Psychosomatics 1971;12:371-379.

22. Liu X, Tang M, Chen K, Hu L, Wang A. The comparison between SDS and CES-D in evaluating depressive symptoms in university students. Chin Ment Health J 1995;9:19-20.

23. Gao J, Tao M. The reliability and validity of Chinese version of revised self-rating anxiety scale (SAS-CR). Chin J Nerv Ment Dis 1994;20:301305.

24. Weissman A, Beck A. Development and Validation of the Dysfunctional Attitude Scale: A Preliminary Investigation. Annual meeting of the American Educational Research Association. Toronto, Ontario, Canada; 1978.

25. Cai L, Zhu X, Peng S, Zhong M, Zhang S. Application of Chinese version of the dysfunctional attitude scale in a sample of Chinese adolescents. Chin J Clin Psychol 2010;18:161-164.

26. Eysenck H. Manul of the Eysenck Personality Questionnaire. London, England: Hodder and Stoughton; 1975.

27. Qian M, Wu G, Zhu R, Zhang X. Eysenck personality questionnairerevised, short scale for Chinese, EPQ-RSC. Acta Psychologica Sinica 2000;32:317-323.

28. Xiao S. Theoretical basis and application of social support rating scale. J Clin Psychiatry 1994;4:98-100.

29. Liu J, Li F, Lian Y. Investigation of reliability and validity of the social support scale. J Xinjiang Med Univ 2008;31:1-3.

30. Shen L, Zhang Y, Liang W. Investigation of child maltreatment: survey among junior school pupils in Henan province of China. Asia Pac Psychiatry 2015;7:85-90.

31. Chan KL. Comparison of parent and child reports on child maltreatment in a representative household sample in Hong Kong. J Fam Viol 2012;27:11-21.

32. Begle AM, Dumas JE, Hanson RF. Predicting child abuse potential: an empirical investigation of two theoretical frameworks. J Clin Child Adolesc Psychol 2010;39:208-219.

33. Tang CS. Corporal punishment and physical maltreatment against children: a community study on Chinese parents in Hong Kong. Child Abuse Negl 2006;30:893-907.

34. Chu DA, Williams LM, Harris AW, Bryant RA, Gatt JM. Early life trauma predicts self-reported levels of depressive and anxiety symp- 
toms in nonclinical community adults: relative contributions of early life stressor types and adult trauma exposure. J Psychiatr Res 2013; 47:23-32.

35. Lundqvist G, Svedin CG, Hansson K. Childhood sexual abuse. Women's health when starting in group therapy. Nord J Psychiatry 2004;58: 25-32.

36. Engert V, Efanov SI, Dedovic K, Dagher A, Pruessner JC. Increased cortisol awakening response and afternoon/evening cortisol output in healthy young adults with low early life parental care. Psychopharmacology 2011;214:26126-26128.

37. Lindert J, von Ehrenstein OS, Grashow R, Gal G, Braehler E, Weisskopf MG. Sexual and physical abuse in childhood is associated with depression and anxiety over the life course: systematic review and meta-analysis. Int J Public Health 2014;59:359-372.

38. Beck AT. Cognitive models of depression. J Cogn Psychother 1987;1:537.

39. Hankin BL, Oppenheimer C, Jenness J, Barrocas A, Shapero BG, Goldband J. Developmental origins of cognitive vulnerabilities to depression: review of processes contributing to stability and change across time. J Clin Psychol 2009;65:1327-1338.

40. Hankin BL. Stability of cognitive vulnerabilities to depression: a shortterm prospective multiwave study. J Abnorm Psychol 2008;117:324-333.

41. Henderson D, Hargreaves I, Gregory S, Williams JM. Autobiographical memory and emotion in a non-clinical sample of women with and without a reported history of childhood sexual abuse. Br J Clin Psychol 2002;41:129-141.

42. Lysaker PH, Meyer PS, Evans JD, Clements CA, Marks KA. Childhood sexual trauma and psychosocial functioning in adults with schizo- phrenia. Psychiatr Serv 2001;52:1485-1488.

43. Allen JG, Coyne L, Huntoon J. Trauma pervasively elevates brief symptom Inventory profiles in inpatient women. Psychol Rep 1998;83: 499-513.

44. Magnus K, Diener E, Fujita F, Pavot W. Extraversion and neuroticism as predictors of objective life events: a longitudinal analysis. J Pers Soc Psychol 1993;65:1046-1053.

45. Talbot NL, Duberstein PR, King DA, Cox C, Giles DE. Personality traits of women with a history of childhood sexual abuse. Compr Psychiatry 2000;41:130-136.

46. Vlasveld MC, van der Feltz-Cornelis CM, Anema JR, et al. The associations between personality characteristics and absenteeism: a crosssectional study in workers with and without depressive and anxiety disorders. J Occup Rehabil 2013;23:309-317.

47. Vranceanu AM, Hobfoll SE, Johnson RJ. Child multi-type maltreatment and associated depression and PTSD symptoms: the role of social support and stress. Child Abus Negl 2007;31:71-84.

48. Tough H, Siegrist J, Fekete C. Social relationships, mental health and wellbeing in physical disability: a systematic review. BMC Public Health 2017;17:414.

49. Poudel-Tandukar K, Nanri A, Mizoue T, Matsushita Y, Takahashi Y, Noda M, et al. Social support and suicide in Japanese men and women - the Japan Public Health Center (JPHC)-based prospective study. J Psychiatr Res 2011;45:1545-1550.

50. Pjescic KD, Nenadovic MM, Jasovic-Gasic M, Trajkovic G, Kostic M, Ristic-Dimitrijevic R. Influence of psycho-social factors on the emergence of depression and suicidal risk in patients with schizophrenia. Psychiatr Danub 2014;26:226-230. 